ISSN: 0213-2087 eISSN: 2444-7080

DOI: https://doi.org/10.14201/shhc202139191208

\title{
CARBÓN, HIERRO Y MAÍZ. ESTUDIO DE UN CASO PRECOZ DE TRUCK SYSTEM EN ESPAÑA: FÁBRICA DE MIERES (ASTURIAS), 1856-1857
}

\section{Coal, iron, and corn. Study of an early case of truck system in Spain: Mieres factory (Asturias), 1856-1857}

David GONZÁLEZ PALOMARES

Universidad de Oviedo

https://orcid.org/0000-0003-0826-2243

Luis Aurelio GONZÁLEZ PRIETO

Real Instituto de Estudios Asturianos

https://orcid.org/0000-0002-2408-2739

Jorge MUÑIZ SÁNCHEZ

Universidad de Oviedo

https://orcid.org/0000-0003-2224-0171

Recibido: 16/01/2021 Revisado: 02/04/2021 Aceptado: 20/04/2021

RESUMEN: El objetivo de este estudio es desvelar los motivos de la implantación del primer economato laboral conocido en Asturias. Para ello se ha recurrido a protocolos notariales, correspondencia custodiada en el Archivo Histórico Nacional de España y en el Archivo Histórico de Asturias, así como fuentes hemerográficas. La conclusión es que a mediados del siglo XIX confluyen en la incipiente industrialización asturiana varios factores que explican el establecimiento de un precoz truck system: la crisis de subsistencias, el reflejo de las teorías filantrópicas y la situación particular de una empresa en estado de necesidad terminan configurando un modelo muy temprano, azaroso y peculiar de economato laboral.

Palabras clave: suministro de alimentos; hambre; minería; metalurgia. 
ABSTRACT: The objective of this study is to reveal the reasons for the implementation of the first company shop known in Asturias. Notarial protocols, correspondence stored in the Archivo Histórico Nacional of Spain and in the Archivo Histórico de Asturias, as well as newspapers have been used for this purpose. The conclusion is that in the middle of the 19th century there are several factors that explain the establishment of an early truck system in the incipient Asturian industrialization: the famine, the philanthropic theories and the particular situation of a company in need form a very early, hazardous and peculiar model of labor company shop.

Key words: food supply; hunger; coal mining; metallurgy.

\section{INTRODUCCIÓN}

Los economatos laborales o tiendas de empresa en Asturias han sido un elemento esencial en el desarrollo de las políticas paternalistas por parte de las compañías mineras e industriales, aunque poco se ha estudiado sobre el origen y la historia de los primeros establecimientos. Siempre que se hace referencia a sus inicios se menciona lo señalado por el ingeniero de Duro y Cía, Francisco Gascue, quien en un trabajo publicado en la Revista Minera en 1883 mencionaba que existían cooperativas de consumo bajo el amparo de las empresas en Mieres y Trubia, así como el economato de la Real Compañía Asturiana de Minas en Arnao ${ }^{1}$. La documentación más antigua que hasta ese momento se manejaba sobre la existencia de un establecimiento de este tipo en Asturias era precisamente la procedente del archivo de esta última, que consistía en un inventario de artículos y databa del año $1874^{2}$.

Durante el proceso de documentación de un trabajo sobre el empresariado minero de mediados del siglo xIx, hemos descubierto -aparentemente traspapelado entre los protocolos de los años 1856 y 1857 del escribano de Mieres Juan Antonio Velasco- un cuadernillo cosido titulado Libro Copiador de Cartas del Almacén de Granos de la Fábrica de Hierros de Mieres, que seguramente era llevado por dicha escribanía. No se trata únicamente del primer economato laboral de Asturias del que se tenga conocimiento -ya que está fechado 18 años antes que la primera referencia aludida en Arnao- sino que estamos también ante la primera tienda de empresa en Asturias en utilizar el truck system o pago en especie al igual que una de las primeras, por no decir la primera, a nivel nacional.

Hemos desarrollado por ello una investigación paralela para averiguar la máxima información posible acerca de este tema. El principal objetivo de este

1. Gascue y Murga, Francisco: "La Industria Carbonera en Asturias (III. El Obrero)», Revista Minera, t. I, n. ${ }^{\circ}$ 26, 1883, pp. 130-131.

2. MuñIZ SÁnChEZ, Jorge: "Paternalismo y construcción social del espacio en el poblado de Arnao (Asturias), 1855-1937», Scripta Nova, vol. IX, n. ${ }^{\circ}$ 249, 2007. 
artículo es, además mostrar este precoz ejemplo, conocer si se trató de un acto de filantropía por parte de la patronal o bien se trató de una artimaña financiera para reducir costes y paliar posibles disturbios. Al mismo tiempo se pretende aclarar si fue un caso coyuntural planificado con mucha más duración. Para ello, en primer lugar, se ha realizado una aproximación general a los sistemas empresariales de truck system y tommy shop con el fin de ofrecer una explicación y exponer su origen y desarrollo a nivel global. Del mismo modo se realiza una introducción a estos sistemas en España, remarcando ya la importancia del caso asturiano a nivel estatal. Para ambas partes se ha acudido a fuentes bibliográficas, consultando estudios tanto generales como particulares del tema. En segundo lugar, comenzamos a adentrarnos en el caso asturiano con una contextualización empresarial, social y económica, vital para comprender la situación y cuáles fueron los factores coyunturales que llevaron a la creación del almacén de granos. Este apartado ha sido reconstruido partiendo de una firme base bibliográfica especificada con fuentes hemerográficas. Por último, el estudio del propio almacén se ha realizado con un exhaustivo análisis del ya citado Libro Copiador de Cartas del Almacén de Granos de la Fábrica de Hierros de Mieres de 1856 y 1857, contrastado y triangulado con la correspondencia de los directivos de la empresa y comerciantes, al igual que con fuentes hemerográficas en los casos que fue posible.

\section{PAGO EN ESPECIE Y DOMINACIÓN EN LA SOCIEDAD INDUSTRIAL}

El truck system -cuyo germen algunos sitúan en la época romana ${ }^{3}$ - consiste en liquidar el sueldo en especie, con las mercancías que produce la empresa u otras necesarias para el sustento diario. Fue utilizado de forma habitual en el siglo Xv para el pago de los asalariados domésticos ${ }^{4}$. Los abusos que se cometieron con este sistema hicieron que en 1512 en Gran Bretaña se tuvieran que tomar medidas legislativas para limitarlo entre el artesanado, ya que el patrón les atribuía un precio superior al del mercado para posteriormente recomprarlos con considerable rebaja ante la incapacidad de los asalariados para venderlos. En muchas ocasiones se realizaba directamente mediante el trueque por alimentos o el uso de viviendas 5 .

Ahora bien, será con el desarrollo industrial, a partir de la segunda mitad del siglo XVIII, cuando el truck system se generalice, sobre todo en la minería ${ }^{6}$. Las

3. LeConte, Antoine: Paiement des Salaires en Nature. Aix en Provence: Thèse pour le Doctorat, Jules Barthélmey Successeur, 1900, pp. 18. Sánchez Domingo, Rafael: «Origen histórico-jurídico del aguinaldo: del strenna romano al salario en especie». En: Campos y Fernández de Sevilla. Francisco Javier (coord.): La Natividad: arte, religiosidad y tradiciones populares. Madrid: Real Centro Universitario Escorial-María Cristina, 2009, pp. 715-729.

4. Lipson, Ephraim: The Economic History of England. London: A. \& C. Black, 1937, pp. 480-482.

5. DoвB, Maurice: Estudios sobre el desarrollo del capitalismo. Madrid: Siglo xxI, 1976, p. 186.

6. Engels, Friedrich: La situación de la clase obrera en Inglaterra. Gijón: Júcar, 1979, 1. ${ }^{a}$ ed. alemana, 1845, pp. 219; Ashton, Thomas Southcliffe: "The Coal-Miners of the Eighteenth Century", 
empresas mineras, al contar por lo general con explotaciones alejadas de los núcleos urbanos, se vieron abocadas a proveer de viviendas a sus trabajadores. Este sistema también se aplicó en algunas industrias que fueron asentadas en zonas alejadas de poblaciones, para aprovechar la fuerza hidráulica de los ríos, debido a lo cual los obreros tenían que recorrer grandes distancias para poder ir al trabajo ${ }^{7}$. Un ejemplo pueden ser las colonias textiles catalanas ${ }^{8}$. A la vez que se les proveía de una casa para vivir, también fue necesario suministrarles los artículos básicos para su sustento, por lo que las empresas empezaron a establecer las conocidas como tommy shop (economatos), que solían ser propiedad de la empresa, pero en bastantes ocasiones también se daban a contramaestres, vigilantes o simplemente comerciantes para su explotación monopolista. Por lo general, estas tiendas fijaban precios abusivos - muy por encima de los de mercado- o daban productos de baja calidad o adulterados, o todo ello a la vez ${ }^{9}$. La adulteración debió de ser frecuente y constituye uno de los elementos centrales del debate entre optimistas y pesimistas en torno al nivel de vida de la clase obrera en la industrialización ${ }^{10}$. En las tommy shop las mercaderías se pagaban entre un 20 y un $25 \%$ más caras que en los comercios libres ${ }^{11}$. Todas estas prácticas tenían como consecuencia una degradación real de las condiciones de vida de la clase trabajadora ${ }^{12}$. Del mismo modo fue señalado por el propio Karl $\operatorname{Marx}^{13}$, haciendo suyas las premisas establecidas por la Children's Employment Commission en 1864.

La propia frugalidad del salario mensual es un factor que empuja al obrero -en déficit crónico- a tomar adelantos que el patrón materializa a través de su tienda, nada competitiva en el mercado libre. Se debe señalar que hay una importante diferencia entre el sistema de expender productos a crédito y el de entregar vales,

Economic Journal, Economic History Suplement, 3, 1928, 26-59; HiLTon, George W.: «The British Truck System in the Nineteenth Century", Journal of Political Economy, 65.3, 1957, 237-256.

7. Gaskell, Peter: The Manufacturing Population of England. London: Baldwin and Gradoc, 1833, pp. 347; Hilton, George W.: "The Truck Act of 1831», The Economic History Review, 10, 1958; RuBIO Álvarez, Alfredo: "Economatos laborales: del truck shop al economato laboral obligatorio", Revista del Trabajo, 3, 1960, p. 63.

8. Dorel-Ferré, Gracia: «Les colonies industrielles catalanes, un patrimoine exceptionnel mais encombrant", Rives Mediterranéennes, 38, 2011, pp. 43-56. Terrades, Ignasi: Les coloniès industrials, un particularisme històric. Barcelona: Laia, 1979.

9. Hilton, George W.: "The British Truck..." p. 246; Navailles, Jean-Pierre: La famille ouvrière dans l' Angleterre victorienne: des regards aux mentalités. Paris: Champ Vallon, 1983, p. 76; GASKELL, Peter: The Manufacturing..., p. 344.

10. BuRNETT, John: Plenty and Want: a social history of food in England from 1815 to the present day. London: Penguin,1968; RuLE, John: Clase obrera e industrialización: historia social de la revolución industrial británica, 1750-1850. Madrid: Crítica, 1990.

11. FIX, Théodore: Notice sur la vie et les ouvrages économiques de M. de Sismondi. Paris: Guillaumin, 1843, p. 53.

12. Marshall, Alfred: Principles of Economics. New York: Cosimo Classics, (primera edición de 1890), 2009, p. 460.

13. MARX, Karl: El Capital. Crítica de la economía política. I. México: Fondo de Cultura Económica, 1978 (1. ${ }^{a}$ ed. alemana 1867), p. 128. 
bonos o jetones (tommy ticket). En el primer caso es el empresario el que hace un crédito al trabajador adelantándole los bienes necesarios para su subsistencia hasta que cobre su salario en moneda corriente, del cual se le descuenta. Tampoco estuvo exento de críticas, porque de alguna manera fomentaba que el obrero se endeudase adquiriendo productos a crédito que luego no podía compensar con su salario, por lo que se convertía en deudor del empresario de por vida y quedaba en una situación de subordinación comparable a la servidumbre ${ }^{14}$. En el segundo, con el pago mediante vales o bonos intercambiables por alimentos, el obrero recibía moneda privada válida únicamente para compras a su patrón y era obligado de este modo a hacer un crédito al mismo, quien no tenía que desembolsar ningún dinero inmediatamente. Esta práctica era, por consiguiente, empleada en algunos casos de falta de liquidez de las empresas.

Al ser las tommy shop las únicas tiendas en las que era admitido este pseudodinero emitido por las propias empresas y, como las familias obreras no contaban con moneda de curso legal para hacer frente a otros gastos corrientes, se llegaba a usar las mercancías más preciadas de los tommy shop para pagar otros bienes o servicios, como a los dentistas ${ }^{15}$. Cuando se les pagaba en dinero de curso legal, en ocasiones se les obligaba -bajo coacción de despido- a aprovisionarse en una tienda de la propia empresa o designada por ella ${ }^{16}$. En la Gran Bretaña victoriana el sistema del truck system era tan corriente que sus perversas consecuencias para las familias obreras fueron magistralmente descritas por Benjamin Disraeli, en su clásica novela Sybil or the two nations.

Pese al gran número de detractores que tuvo el truck system, los empresarios y algunos moralistas lo defendían porque consideraban que los obreros, en la mayoría de los casos, despilfarraban sus emolumentos en moneda de curso legal bebiendo en las tabernas, dejando a la familia sin dinero para su sustento ${ }^{17}$. Sin duda este tipo de apreciación resulta muy sesgada, como la idea concomitante de que el consumo de alcohol indisponía al minero para el trabajo -lo cual hubiera sido un suicidio económico e incluso físico en el sentido literal- por lo que hay que concluir que normalmente se bebía de forma calculada -aunque no

14. Bonneff, Léon y Bonneff, Maurice: La vie tragique des travailleurs. Paris: Études et Documentation Internationales, 1984 (1. ${ }^{a}$ ed. 1908), p. 51; BABBAGE, Charles: On the Economy of Machinery Manufactures. London: Charles Knight, 1832, p. 545.

15. BabBage, Charles: On the Economy..., pp. 548-549.

16. BonnefF, Léon y BonnefF, Maurice: La vie tragique..., p. 102.

17. Sobre el alcoholismo de las clases obreras vid. Villermé, Louis-René: Tableau de l'état physique et moral des ouvriers employers dans les manufactures de coton, de laines et de soie. Paris: Jules Renouard et Cie, 1840, p. 36; SIERra Álvarez, José: "¿El minero borracho? Alcoholismo y disciplinas industriales en Asturias», Cuadernos del Norte, n. ${ }^{\circ} 6$ (29, 1985, p. 60, señala que «economatos y cooperativas, a través de la forma de pago de los géneros -libretas o bonos- constituían también medios indirectos de inculcación de hábitos antialcohólicos». Este tema también es tratado en CAMpos Marín, Ricardo: Alcoholismo, medicina y sociedad en España (1876-1923). Madrid: Consejo Superior de Investigaciones Científicas, 1997, p. 177. 
necesariamente moderada- para evitar grandes riesgos ${ }^{18}$. Por lo general, este tipo de cristalizaciones ideológicas fueron de la mano con la articulación de dispositivos paternalistas más generales -algo lógico pues su base es la supuesta necesidad de tutela del obrero- que tenían en la provisión de viviendas el centro de un artefacto ideado para moldear al trabajador y su familia ${ }^{19}$. Lo que no señalaban estos teóricos de la moralidad era lo que tenían las tabernas de zona opaca al poder casi omnímodo de la empresa minera, donde se sumaban sociabilidad espontánea, ocio no intervenido y eventualmente autoorganización obrera. Eran un lugar franco para los trabajadores ${ }^{20}$. Todo ello lo recoge Zola en su novela ampliamente documentada en hechos reales Germinal, donde Rasseneur, el tabernero, es un antiguo minero despedido por sus actividades reivindicativas y su establecimiento el corazón de la comunidad minera y, por extensión, de los conflictos laborales.

Sea como fuere, la abolición del truck system será una de las peticiones más clamorosas del incipiente movimiento obrero. Será una de las principales reivindicaciones de la Segunda Internacional junto a la jornada de ocho horas ${ }^{21}$. Los gobiernos europeos se vieron impelidos a tomar medidas para limitarlo o prohibirlo. Gran Bretaña inició esa senda con la aprobación en 1831 de la Truck Act, que pretendió abolirlo aunque siguió siendo una práctica muy corriente, ya que en numerosos condados los jueces estaban al servicio de los empresarios ${ }^{22}$. Debido a su ineficacia, la Truck Act fue seguida de otras disposiciones en los años 1851 y 1871, que poco a poco acabaron con esta práctica ominosa ${ }^{23}$. Prusia prohibió esta práctica en 1849. En Bélgica fue la Ley de 16 de agosto de 1887 la que la proscribió parcialmente. En Francia, entre 1895 y $1910^{24}$. En España, donde este tipo de establecimiento será más conocido como "cantina", será prohibido por el Real Decreto de 18 de julio de 1907, aunque también siguió aplicándose por algunas empresas,

18. Vulic, Milan: Le cabaret, le bistrot, lieu de la sociabilité populaire dans le bassin houllier du Nord-Pas-de-Calais (1750-1985). Lille: Thèse de doctorat, Université Lille 3, 1991, p. 407.

19. CAmpos Marín, Ricardo: «Casas para obreros: un aspecto de la lucha antialcohólica en España durante la Restauración", Dynamis, n. ${ }^{\circ}$ 14, 1994, pp. 111-130.

20. Sierra Álvarez, José: El obrero soñado. Ensayo sobre el paternalismo industrial (Asturias, 1860-1917). Madrid: Siglo Xxi, 1990, pp. 37-39; SERrano, Carlos: «Le vin du proletaire. Alcool et sociabilite ouvriere en Espagne à la fin du XIxe siècle». En Carrasco, Rafael (coord.): Solidarités et sociabilités en Espagne (XVI-XX siecles). Besançon: Centre de recherches sur l'Espagne moderne, 1991, pp. 371-389; URía GonZÁLEZ, Jorge: "La taberna: un espacio multifuncional de sociabilidad popular en la Restauración española", Hispania: Revista española de historia, n. ${ }^{\circ} 214$, 2003, pp. 571-604; CoOper-Richet, Diana: Le peuple de la nuit. Mines et mineurs en France (XIXe-XXIe siècle). Paris: Perrin, 2002, p. 199.

21. Azcárate, Gumersido de: "Leyes obreras, leyes sociales o leyes del trabajo", Revista de España, n. ${ }^{\circ} 144,1894$, pp. 54-80.

22. Hilton, George W.: "The Truck...». pp. 470-479; Philips, David: "The Black County Magistracy 1835-60. A Changing Elite and the Exercise if this Power", Midland History, n. ${ }^{\circ}$ 3, 1976, p. 167; Hilton, George W.: "The British Truck...», p. 237.

23. Engels, Friedrich: Die Lage der Arbeitenden Klasse in England. Zweite Stuttgart: Auflage, 1892, p. 11

24. CaPitant, Henri: Note sur la législation française tendant à réprimer le Truck-System. Paris: Association Nationale Française pour la Protection Légales des Travailleurs, 1912. 

EN ESPAÑA: FÁBRICA DE MIERES (ASTURIAS), 1856-1857

a pesar de la claridad con que estaba formulado el decreto: «deberá prohibirse el pago con pagarés, vales, cupones o en cualquier otra forma que se considere representativa de la moneda de curso legal».

\section{EL TRUCK SYSTEM EN ESPAÑA}

En nuestro país, las referencias más antiguas del truck system provenían de las minas andaluzas y murcianas y datan del último tercio del siglo xix. Uno de los primeros en dar testimonio de este sistema fue el geógrafo francés Casimir Delamarre, quien explicará que la forma más habitual de explotar el plomo en la sierra de Almagrera era la varada, por la que se liquidaban los salarios cada tres mensualidades. Como los obreros no podían subsistir tanto tiempo por ellos mismos, la empresa les proveía de comida -principalmente garbanzos- que se descontaba del salario. También podían adquirir ropas y otros objetos a crédito en las tiendas de la empresa a precios bastante más elevados que los del mercado ${ }^{25}$. Esta práctica habitual del truck system en la Sierra de Almagrera se agravó de forma sustancial en el último tercio del xix como forma encubierta de bajada de salarios ${ }^{26}$. La utilización de este peculiar sistema de pago también se dio con mucha frecuencia en las minas de Linares, así como en las de La Unión, en Murcia ${ }^{27}$. Por su parte, Río Tinto Company Limited creaba en Huelva, en el año 1878, un almacén de productos de primera necesidad ${ }^{28}$.

Ahora bien, será en el periodo de entresiglos cuando el truck system se empiece a percibir como un problema social relevante a causa de la acción de los primeros grupos de obreros organizados, porque -como señala Adolfo A. Buyllasu abolición es una de las reivindicaciones más corrientes de los importantes movimientos huelguísticos que tuvieron lugar en la minería vizcaína en estos años ${ }^{29}$.

25. Delamarre, Casimir: «La province d'Almeria économique et sociale», Bulletin de la Société de Géographie de Paris, XIV, 1867, p. 53.

26. SÁnchez Picón, Andrés: La minería del Levante almeriense, 1838-1930. Especulación, industrialización y colonización económica. Almería: Cajal, 1983, p. 242; PÉrez DE Perceval, Miguel Ángel: La minería almeriense contemporánea (1800-1930). Almería: Zegel, 1989, p. 160.

27. Pérez de Perceval, Miguel Ángel: La minería almeriense..., p. 165; Martínez Carrión, José Miguel: Historia económica de la Región de Murcia, siglos XIX y XX. Murcia: Consejería de Educación y Cultura, 2002, p. 263; EgEa Bruno, Pedro María: «El Movimiento Obrero en la Sierra de Cartagena (18751923)", Anales de H. ${ }^{a}$ Contemporánea, n. ${ }^{\circ}$ 5, 1986, pp. 130; Vilar, Juan Bautista; EgEA, Pedro y Moreno, Diego: El Movimiento Obrero en el Distrito Minero de Cartagena-La Unión. Murcia: Academia Alfonso X El Sabio, 1986, p. 164; Egea Bruno, Pedro María: El distrito minero de Cartagena. En torno a la primera guerra mundial (1909-1923. Murcia: Universidad de Murcia-Ayuntamiento de Cartagena, 1986, p. 290.

28. Arenas Posadas, Carlos: Empresa, mercados, mina y mineros. Tío Tinto, 1873-1936, Huelva: Universidad de Huelva, 1999, p. 275; PAz SÁnchez, José Juan de: Entre el puerto y la mina. Antecedentes del movimiento obrero organizado en Huelva (1870-1912). Huelva: Universidad de Huelva, 2014, p. 141 .

29. Álvarez Buylla, Adolfo: "Las huelgas en España", La Lectura. Revista de Ciencias y de Artes, n. ${ }^{\circ} 1,1901$, p. 12. 
La primera huelga importante en la que consta esta demanda fue la que llevaron a cabo los mineros de Triano-Somorrostro, en el mes de mayo de 1890. La chispa fue el despido de cinco trabajadores en la compañía minera Orconera por haber organizado los actos del $1 .^{\circ}$ de mayo, lo que desencadenó un gran movimiento de solidaridad. Entre las reivindicaciones estaba la «supresión absoluta de los cuarteles o barracones, dejando, por tanto, en completa libertad los trabajadores para que se administren de comestibles donde lo crean conveniente 30 .

Si Disraeli mostraba las estampas del sistema de tommy shops británicas, será Ramiro Pinilla quien en su novela Verdes valles, colinas rojas retrate la cruda realidad del truck system de las minas de Vizcaya. Por su parte, Olga Macías Muñoz ${ }^{31}$ constata que las tiendas de las zonas mineras eran un monopolio, por el cual los comerciantes pagaban suculentas cantidades de dinero a los propietarios de las minas y en las que se daba participación en la gestión a los propios capataces. La huelga finalizó con el famoso pacto firmado con mediación del general Loma ${ }^{32}$. Pese a su prohibición, los capataces -que obtenían suculentos beneficios de ellasse las ingeniaron para que los mineros tuvieran que seguir utilizándolas para abastecerse bajo coacción. Por este motivo, en la huelga de 1903 la supresión de las cantinas volverá a ser reivindicada y obtenida ${ }^{33}$.

Por todo ello, podemos decir que el truck system fue una práctica muy extendida por las principales comarcas mineras de nuestro país. Es sorprendente, pues, que la historiografía asturiana no haya aportado una sola referencia a este sistema de pagos de salario, en especial existiendo ejemplos de numismática minera, es decir, de acuñaciones de monedas que solo se podían utilizar en los economatos o cooperativas de las empresas ${ }^{34}$. Tampoco el estudio de los economatos parece haber retenido demasiado la atención de la historiografía asturiana, ya que las referencias que encontramos suelen ser tangenciales y dentro del estudio genérico de las prácticas de paternalismo industrial ${ }^{35}$. Desde finales del siglo XIx hasta los años ochenta del siglo xx -pero especialmente durante el franquismo- fueron un factor

30. Sanz y Escarpín, Eduardo; Puyol y Alonso, Julio y Salillas, Rafael: Informe referente a las minas de Vizcaya. Madrid: Instituto de Reformas Sociales, 1904, p. 32.

31. Macías MuÑoz, Olga: "La alimentación de los mineros de Triano (Vizcaya): Cantinas y tiendas obligatorias, 1882-1899", en Jornadas Científicas Minería y desarrollo empresarial en España, Almadén, 17 y 18 de febrero de 2005.

32. Tuñón de LaRA, Manuel: El movimiento obrero en la historia de España (I). Madrid: Sarpe, 1985, p. 297; Fusi Aizpurua, Juan Pablo: Política obrera en el País Vasco [1880-1923]. Madrid: Turner, 1975; Miralles, Ricardo: «Vizcaya en huelga: mayo de 1890 en las minas». En: Montero, Manuel (coord.): Historia de los montes de hierro (1840-1960). Bilbao: Museo de la Minería del País Vasco EdicionesBeta, 2004, p. 30; Hidalgo García, Sara "Emociones socialistas en la huelga minera de 1890. La formación de la conciencia de clase y el giro emocional", Historiográficas, n. ${ }^{\circ}$ 10, 2015, pp. 37-38.

33. Sanz y Escarpín, Eduardo; Puyol y Alonso, Julio y Salillas, Rafael: Informe... pp. 34-46.

34. Calvo Rebollar, Miguel: "Una introducción a la numimástica minera», XII Congreso Internacional sobre patrimonio geológico y minero. Boltaña, 2012, pp. 39-66.

35. Muñiz SÁnchez, Jorge: Del pozo a casa. Genealogías del paternalismo minero contemporáneo en Asturias. Gijón: Trea, 2007. 

EN ESPAÑA: FÁBRICA DE MIERES (ASTURIAS), 1856-1857

amortiguador de las duras condiciones sociales ${ }^{36}$. Además, se trató de un canal de comercialización que en Asturias llegó a alcanzar cuotas superiores al 10 \% del mercado ${ }^{37}$. Esto va en consonancia con los economatos laborales surgidos de la filantropía empresarial del siglo XIX, que buscaba fidelizar al obrero, generando buena imagen patronal y ejerciendo un papel moderador sobre las expectativas salariales en metálico de la plantilla ${ }^{38}$. Esto era perfectamente compatible con las ideas del socialismo utópico que en esta época penetraron incluso en círculos industriales patronales y de dirección. Bastantes alumnos de las escuelas francesas de ingeniería abrazaron esta ideología al ser captados por el grupúsculo encabezado por Prosper Enfantin y Saint-Armand Bazard. En las filas de los ingenieros próximos al saint-simonismo militarán personajes como los hermanos Émile e Isaac Pereire, Eugène Flachat y el financiero Adolphe D'Eichthal, estos dos últimos muy relacionados con el grupo Riánsares-Lillo-Grimaldi, detentador de la mitad del capital de la Compagnie Minière et Métallurgique des Asturies, propietaria de la Fábrica de Mieres en el periodo aquí estudiado. Eugéne Flachat era el ingeniero jefe de Chemins de Fer de l'Ouest e ingeniero consultor de los Ferrocarriles del Norte de España, fue el asesor técnico del grupo Riánsares-Lillo-Grimaldi en la construcción del ferrocarril de Langreo y participó junto con los ingenieros Juncker y Sauvage en la elaboración del informe y evaluación de todos los activos del grupo Riánsares-Lillo-Grimaldi en 1853. En cuanto a Adolphe D' Eichthal era socio del duque de Riánsares en la Empresa Carbonera de Langreo y Siero y en 1854, con motivo de la ascensión al poder de los progresistas por la Vicalvarada, se convirtió en el testaferro de los créditos que el grupo Riánsares-Lillo-Grimaldi tenía contraídos con la Compañía del Ferrocarril de Langreo ${ }^{39}$.

\section{CONTEXTO EMPRESARIAL, SOCIAL Y ECONÓMiCO}

Para comprender la necesidad de creación del almacén de granos por parte de la Fábrica de Hierros de Mieres en 1856 es de vital importancia conocer el estado socio-económico. La población mierense sufría una fuerte crisis de subsistencia que, sumada a la difícil situación económica que atravesaba la empresa, hacía que los trabajadores se encontrasen en un pésimo estado.

La conocida como Fábrica de Mieres había sido creada en 1844 por la sociedad Asturias Mining Company o Anglo-Asturina, con mayoría de capital británico, por lo que sus acciones cotizaban en la bolsa de Londres. Se trataba de una

36. Benito Del Pozo, Carmen: La clase obrera asturiana durante el franquismo. Madrid: Siglo XXI, 1993, p. 263.

37. Stern, Louis W.; El-Ansary, Anne y Cruz Roche, Ignacio: Canales de Comercialización. Madrid: Pearson Education, 1999, p. 232; Rubio Álvarez, Alfredo "Economatos laborales...», p. 63.

38. Sierra Álvarez, José: El obrero soñado..., pp. 77-95.

39. Ratcliffe, Barrie M.: "Les Pereire et le Saint-simonisme», Économies et Sociétés, n. ${ }^{\circ}$ 5, 1971, pp. 28-41; Le Bret, Hervé: Les frères d'Eichthal. Paris: Presses de l'Université París-Sorbonne, 2012. 
sociedad que se dedicó a la explotación de minas de hierro y carbón, así como construyó una siderurgia integral a la inglesa, en la que se consiguió obtener por primera vez arrabio utilizando coque en nuestro país.

La compañía no conseguirá beneficios por lo que cinco años después de su constitución arrastraba una situación financiera deplorable, acumulando pérdidas por valor de 4.969.128 reales. Mediante Real Orden de 16 de agosto de 1849 el gobierno decretó su disolución, por no respetar la legislación de sociedades españolas, así como por las pérdidas acumuladas.

Esta situación será aprovechada por el grupo financiero encabezado por el duque de Riánsares, marido morganático de la reina madre M. ${ }^{a}$ Cristina, que conseguirá adquirir a través del banquero León Lillo el 50 \% de la disuelta compañía por 2.000.000 de reales de vellón en 1850. Tres años después se constituirá una nueva sociedad comanditaria por acciones con un capital de 4.000.000 de francos, repartido en 16.000 acciones, en la que el grupo de Riansares detentará la mitad del capital y la otra será propiedad de los antiguos accionistas de la Asturian Mining Company ${ }^{40}$, con la razón social Juan Grimaldi y Cía. y bajo la denominación de Compagnie Minière et Métallurgique des Asturies.

La nueva compañía no conseguirá los capitales necesarios para acometer las inversiones requeridas para mejorar $s$ rentabilidad y conseguir los ansiados beneficios, por lo que su situación financiera continuará siendo crítica. A principios de 1855, se nombra como Director a Edward Fettyplace, que había dirigido previamente los establecimientos metalúrgicos de Córcega, cerca de Bastia, pertenecientes a la Société Industrielle de la Corse ${ }^{41}$.

El nuevo director tiene que hacer frente a una situación financiera muy complicada, agravada por la nueva legislación ferroviaria de ese año 1855, que en su artículo 20 suprime el pago de aranceles del material ferroviario que se importe para la construcción de ferrocarriles ${ }^{42}$, dejando a la factoría mierense completamente al margen por sus carencias técnicas y de competitividad respecto a la siderurgia extranjera. No terminado el año 1855, todavía se agrava más aún la situación, ya que el nuevo proyecto de aranceles proponía rebajar en gran medida el impuesto para todos los productos siderúrgicos, obligando a Edward Fettyplace (1856) a escribir un memorándum en defensa de la industria ferrera española. Todo esto unido a las crónicas desavenencias existentes entre el grupo Grimaldi-Riánsares y los accionistas británicos, llevó a la decisión de enajenar mediante pública subasta la compañía. A la primera puja -el 16 de diciembre de 1856 en la notaría de Fremyn en París y con un precio de salida de 2.250.000 francos- no concurrió ningún interesado, por lo que se convocó una segunda para el 17 de marzo de 1857, con una rebaja de 100.000 francos en el precio de salida. Es decir, en los meses finales de 1856 y

40. González Prieto, Luis Aurelio: Oro negro, dinero sucio. Oviedo: Laria, 2017, pp. 148-155.

41. Journal du génie civil, des sciences et des arts, vol. n. ${ }^{\circ}$ 13, 1846, p. 503.

42. ARTOlA, Miguel: "La acción del Estado: la aportación estatal a la construcción». En: Artola, Miguel: Los ferrocarriles en España 1844-1943. Madrid: Banco de España, 1978, p. 369. 
comienzos de 1857 la situación económica de la empresa es absolutamente crítica y lo más probable es que tuviese una necesidad crónica de todo tipo de capital, pero en concreto de circulante necesario para pagar los insumos imprescindibles (salarios, materias primas, transportes, etc.) para continuar produciendo.

Como decíamos, la situación de crisis no era exclusiva de la empresa. Según recogía en su Manifiesto del Hambre el VIII marqués de Camposagrado, José María Bernaldo de Quirós y Carreño (1856), la cosecha de 1852 se había perdido completamente en el Occidente asturiano. Al año siguiente, en toda la provincia solamente se había recogido un cuarto de lo habitual, insuficiente para compensar la escasez arrastrada de años anteriores. Parece ser que el maíz fue diezmado por las desfavorables condiciones que hicieron elevar los precios de una forma alarmante. A la vez, las fuertes nevadas del invierno volvieron infranqueables los puertos cantábricos, lo que imposibilitó la importación de granos de Castilla y, además, el puerto de Gijón estaba cerrado a los barcos provenientes de Galicia por el brote de cólera allí surgido. El hambre se extendió por toda la provincia y facilitó la penetración de la epidemia de cólera, causando una gran mortalidad entre la población mal nutrida ${ }^{43}$.

A principios del verano la situación era tal que el marqués de Camposagrado lanzó el ya mencionado Manifiesto del Hambre, en el que reflejaba la grave situación de la región y solicitaba del gobierno las ayudas necesarias para paliarla. El Manifiesto fue publicado en el diario El Industrial, pero la mayoría de los ejemplares fueron secuestrados por orden del gobernador civil J. de los Santos, a la vez que sancionados. No obstante, este consiguió difundirlo mediante la impresión de hojas sueltas. Días después, con motivo de los eventos revolucionarios de ese verano de 1854, el Marqués de Camposagrado presidió la Junta Revolucionaria de la Región, pese a ser miembro del Partido Moderado, y tuvo que dar refugio al mismo gobernador civil en su casa, para librarlo de una muerte segura a manos de las masas populares ${ }^{44}$.

En el año 1855, la peste se cebó en toda la región y en particular en el concejo de Mieres y, además, la cosecha obtenida en los campos asturianos no compensó las carencias producidas por las anteriores ${ }^{45}$. Así, a finales de 1855 el gobernador de Asturias por aquel entonces, Antonio Romero Ortiz, alertaba de la insuficiencia de las cosechas e indicaba que la guerra en Crimea cerraba la posibilidad de importar de Rusia los cereales necesarios, por lo que los precios en nuestro país se encarecerían más aún ${ }^{46}$. La situación era tan extrema que la fanega de centeno llegará a pagarse junio de 1856 a 110 reales -que como decía La Esperanza «es casi

43. Moro Barreñada, José María: Las epidemias de cólera en la Asturias del siglo XIX. Oviedo: Universidad de Oviedo, 2003, p. 73.

44. García García, Carmen: «Liberalismo y revolución burguesa en Asturias (1833-1868)». En Historia de Asturias. Oviedo: Editorial Prensa Asturiana, t. III, 1990, pp. 645-646.

45. La Nación, n. ${ }^{\circ} 2248,30-9-1855$.

46. Según La Nación, n. . 2345, 22-1-1856, faltaban en Asturias 30.075 fanegas de escanda, 68.937 de trigo, 23.619 de centeno, 586.521 de maíz, 25.735 de habas y 191.583 de patatas. 
el triple del precio más alto jamás alcanzado»- y cuando en el mercado mayorista de cereales de Zamora rondaba los $32^{47}$.

Para colmo de males, en junio -cuando el maíz ya se encontraba sembradose produjeron unas terribles inundaciones en toda la provincia, echando a perder la mayor parte de la cosecha ${ }^{48}$. La especulación con los alimentos alcanzó extremos aberrantes. La mayoría de las veces los labradores no llegaban con sus granos a los mercados, porque los especuladores les abordaban en los caminos para comprarles todo y revenderlo mucho más caro ${ }^{49}$. El gobierno se vio obligado, el 21 de noviembre de 1856, a autorizar al Ministerio de Hacienda para que procediera a la compra de los cereales necesarios para nivelar los precios ${ }^{50}$. A principios de enero de 1857 el gobierno ya tenía almacenadas unas 17.000 fanegas de trigo en el puerto de Málaga, si bien, según refleja la prensa, era de pésima calidad y no servía para contener el aumento de los precios del pan ${ }^{51}$.

\section{El Almacén de Granos de la Fábrica de Mieres: ¿Filantropía o financiación DEL CAPITAL CIRCULANTE DE LOS SALARIOS?}

A principios del mes de diciembre de 1856 la situación por la que estaban pasando los trabajadores de la Fábrica de Hierros de Mieres era dramática debido a la pésima situación de la empresa y la fortísima crisis de subsistencia. Las pagas en la Fábrica se realizaban a mes vencido, lo que hacia que muchos trabajadores no pudiesen llegar a fin de mes. Se veían obligados a pedir adelantos a la empresa, que no eran concedidos y por lo tanto acudían a los tenderos para que los fiasen hasta el día de paga, pero con altos precios e intereses. Para poder subsanar en parte esta situación, el director Edward Fettyplace tomó la resolución de comprar alimentos de primera necesidad para venderlos «a los precios a que líquidamente resulten puestos en el almacén" "52. Así, los comestibles serían vendidos al precio que habían sido comprado, teniendo en cuenta también los costes de transporte y el salario de los trabajadores encargados del almacén. Los destinatarios eran un número elevado, puesto que en ese año la cifra aproximada de empleados de la

47. La Esperanza, n. ${ }^{\circ}$ 3.588, 30 de junio de 1856. Gaceta de los Caminos de Hierro, n. ${ }^{\circ}$ 15, 3 de agosto de 1856.

48. La España, n. ${ }^{\circ} 2514,10$ de junio de 1856.

49. Moro Barreñada, José María: Las epidemias de..., pp. 78.

50. Circular n. ${ }^{\circ}$ 362, Gaceta de Madrid, n. ${ }^{\circ}$ 1418, 21 de noviembre de 1856; Artículo del Centinela de Asturias, transcrito por La Iberia, 16 de enero de 1856.

51. Gaceta de los Caminos de Hierro, n. ${ }^{\circ}$ 4, 25 de enero de 1857. Podemos decir que la Gaceta de los Caminos de Hierro era la prensa salmón de la época.

52. Carta del director Fettyplace al gobernador civil de la provincia, 13 de diciembre de 1856. Archivo Histórico de Asturias (AHA), Oviedo-España, Protocolos del escribano de Mieres Juan Antonio Velasco (en adelante, PJAv), caja 10.664. 

EN ESPAÑA: FÁBRICA DE MIERES (ASTURIAS), 1856-1857

Compagnie Minière et Métallurgique des Asturies era cercana a los 900 trabajadores, de los que unos 400 trabajaban directamente en la factoría siderúrgica ${ }^{53}$.

Las palabras de Fettyplace muestran una aparente actitud filantrópica por parte de la empresa, que intentaba ayudar desinteresadamente a sus trabajadores. Es más, la utilización en fecha tan temprana del término clase obrera nos hace pensar que, al igual que bastantes ingenieros franceses, este británico podría estar influido por los pensamientos de los socialistas utópicos. No es descabellado pensar que Flachat o el mismo Adolphe D' Eichthal, muy familiarizados con esta ideología, hubiesen sido sus mentores para que Grimaldi le nombrase director de los activos de la Compagnie Minière et Métallurgique des Asturies $^{54}$.

Si bien la idea del proyecto pudo provenir del altruismo patronal, todo cambia cuando fue llevado a la práctica. Con su puesta en marcha se estable un sistema de vales a crédito. Si los trabajadores necesitaban un adelanto para alimentos acudían al almacén. El gerente contabilizaba a nombre del trabajador la cantidad adquirida y posteriormente enviaba una relación al contador de la Fábrica de Mieres para que dedujera las cantidades adquiridas de los salarios ${ }^{55}$. Es decir, sin ninguna duda estamos ante un truck system. Aunque los trabajadores no estaban obligados a acudir al almacén de la empresa, su pésima situación hacía que se viesen forzados a aceptar esos vales de crédito para el almacén y por ende la posterior reducción de sus jornales en dinero.

Según el director Fettyplace, el sistema truck system había sido establecido en el almacén para proteger al obrero de posibles abusos que cometiera el encargado. Esto da a entender que, posiblemente el gerente del almacén, Ramón Martínez y sus trabajadores alterasen los precios y maquilasen los granos a su antojo de forma habitual para su propio beneficio. De ese modo, al establecer ese «trueque de sudor humano por pan" se suprimía el uso de efectivo, eliminando las posibilidades de hurto y enriquecimiento del tendero.

Se trataba de un elemento novedoso en la región que podía desencadenar ciertos conflictos. Es por ello por lo que, el 13 de diciembre de 1856, Fettyplace envía una misiva al Gobernador Civil, máxima autoridad política de la provincia, con la intención de recabar su autorización para el establecimiento. Se temía que fuese visto como una competencia desleal por parte de "los traficantes de granos" y desatase una fuerte reacción contra el establecimiento, por lo que el apoyo institucional era de vital importancia ${ }^{56}$. El mismo día el encargado del Almacén de Granos de la Fábrica, Ramón Martínez, envió una carta al mencionado marqués

53. La Época, n. ${ }^{\circ}$ 2.659, 21 de agosto de 1858, durante la visita de la Reina Isabel II a Mieres fija el número de empleados de toda la Compañía en 860. Juan de Dios de la Rada (1860) estima en 400 los operarios de la Fábrica cuando la Reina la visita en el verano de 1858.

54. González PRieto, Luis Aurelio: Oro negro..., pp. 148-155.

55. Libro Copiador de Cartas del Almacén de Granos de la Fábrica de Mieres, carta del 17 de agosto de 1857, AHA, PJAV, caja 10.664, cuadernillo aparte.

56. Carta de Fettyplace al Gobernador Civil de la provincia, 13 de diciembre de 1856, AHA, PJAV. 
de Camposagrado para que intercediera ante el gobernador con vistas a la pronta apertura $^{57}$.

Sin embargo, el inicio de las actividades del almacén tuvo lugar antes incluso. Así, el 7 de diciembre el administrador de la Fábrica de Hierros, Sr. Payne, solicitó al comerciante Vicente Díaz de Gijón -que a la vez era el encargado del almacén de hierros de la propia empresa en esta localidad, lo que es muy significativo por enlazar muy directamente con la tradición de intermediación de empleados en el truck system-, la compra de 300 fanegas de maíz y 100 de trigo con la intención de venderla sin ganancia a los obreros ${ }^{58}$. Cuatro días después, el 11 de diciembre, se insiste a Díaz en que tanto el maíz como el trigo fuera el más barato posible, no haciéndose ninguna referencia a la calidad de los artículos ${ }^{59}$. El primer cargamento que llegó al Almacén de Granos fue de 120 fanegas de trigo a 90 reales a mediados del mes de diciembre. En vista de las fiestas navideñas, se hizo acopio, por lo que se adquirieron 300 fanegas de maíz a 53 reales cada una, otras 100 de trigo a 90 y 32 arrobas de arroz a 27 reales. Por lo general, estos serán los tres tipos de granos que se pongan a disposición de los obreros, aunque en algunas ocasiones también se adquirieron importantes cantidades de cebada.

Tabla 1. Cargamentos, precios y gastos de diciembre de 1856

\begin{tabular}{|l|l|l|l|}
\hline \multicolumn{1}{|c|}{ CEREAL } & \multicolumn{1}{|c|}{ CANTIDAD } & \multicolumn{1}{c|}{ PRECIO POR CANTIDAD } & \multicolumn{1}{c|}{ COSTE TOTAL } \\
\hline Maíz & 300 fanegas & 53 reales por fanega & 15.900 reales \\
\hline Trigo & 220 fanegas & 90 reales por fanega & 19.800 reales \\
\hline Arroz & 32 arrobas & 27 reales por arroba & 864 reales \\
\hline Totales & 520 fanegas y 32 arrobas & - & 36.564 reales \\
\hline
\end{tabular}

El transporte de los cereales desde Gijón se hacía en los carros que retornaban de llevar los hierros producidos por la propia fábrica, con un coste de cuatro reales para las fanegas y de uno para las arrobas ${ }^{60}$. No obstante, con bastante frecuencia llegaban las mercancías en malas condiciones y con importantes mermas. En este sentido, el 23 de marzo de 1857, el encargado del almacén encarecía a Vicente Díaz que se ocupase de comprobar personalmente la medida exacta de grano que traía cada carro, que tomara nota y la enviara con el propio cargamento ${ }^{61}$. Pese a

57. Carta del director del Almacén de Granos de la Fábrica de Hierros al Marqués de Camposagrado, 13 de diciembre de 1856, AHA, PJAV.

58. Carta a Vicente Díaz, 7 de diciembre de 1856, AHA, PJAV.

59. Carta a Vicente Díaz, 11 de diciembre de 1856, AHA, PJAV.

60. Carta a Vicente Díaz, 23 de diciembre de 1856, AHA, PJAV.

61. Carta a Vicente Díaz, 18 de febrero de 1857, AHA, PJAV. 
las medidas que se tomaron para evitar los hurtos por parte de los carreteros, el 23 de julio de 1857 Ramón Martínez se quejaba a Vicente Díaz de que la última remesa de maíz había venido muy mal acondicionada y faltaba una fanega ${ }^{62}$. Otro problema por el que se recibieron muchas quejas fue la calidad de los géneros. El 8 de agosto de 1857 Ramón Martínez mandó varias cartas a Vicente Díaz por este motivo, llegando incluso a suspender el envío de cinco carros con 89 fanegas de maíz por su pésima calidad ${ }^{63}$. Tampoco fue fácil la logística. Las primeras papeletas o vales que se expidieron en las oficinas de la empresa para que los obreros retiraran los granos del almacén establecían los sábados para hacerlo, lo que producía importantes aglomeraciones esos días, por lo que el encargado recomendó a las oficinas de administración de la empresa que no fijaran un día concreto ${ }^{64}$.

Sin embargo, pronto y probablemente como consecuencia de las dificultades que atravesaba la empresa, unidas a los problemas específicos del economato que se vienen detallando aquí, se abandonaron las primigenias intenciones de vender los cereales a los trabajadores al precio más bajo posible, ya que el 17 de agosto de 1857 el encargado del Almacén de Granos pasó una nota a la administración de la empresa para que realizaran los correspondientes descuentos en los salarios mensuales a los trabajadores por las cantidades de cereales recogidas en el mes pidiendo una rebaja en los precios ${ }^{65}$. La causa son las continuas protestas de los obreros. Si los precios de la fanega de trigo rondaban los 100 reales a comienzo del año 1857 en el puerto de Gijón, tenemos conocimiento de que a principios de abril llegó una gran cantidad de este grano adquirido por el gobierno que se vendió a entre 58 y 64 reales, pero esta bajada de precios considerable no se reflejó en los descuentos que en esos meses se hacían a 96 reales la fanega ${ }^{66}$. Hay que señalar también que a principios del mes de agosto llegaron a la comarca cereales de la nueva cosecha procedentes de Castilla a precios muy buenos. En una carta del 25 agosto de 1857, del encargado del almacén al director de la fábrica, se explica el malestar que había entre los obreros, ya que en el mercado se estaba vendiendo el copín de trigo bueno de castilla a 8 1⁄2 reales, es decir 2 1⁄2 más barato de cómo lo descontaba la empresa a principios de agosto ${ }^{67}$. Además, se constataba que los trabajadores de la compañía se quejaban de que la mayoría de las veces solo había maíz disponible en el almacén. Es más, un día después se volvía a comentar sobre los precios del trigo y del maíz en los mercados asturianos y se precisaba que la

62. Carta a Vicente Díaz, 23 de julio de 1857, AHA, PJAv.

63. Cartas a Vicente Díaz, 23 de julio y 8 de agosto de 1857, AHA, PJAV.

64. Carta a la Oficina de la Empresa, 14 de enero de 1857, AHA, PJAV.

65. Carta al director de la Fábrica, 17 de agosto de 1857, AHA, PJAv. En el real se había introducido el sistema decimal en 1848 A.H.A.

66. La Época, n. ${ }^{\circ}$ 5, 4 de abril de 1857. Los precios del trigo del gobierno a principios del mes de agosto rondaban los 40 reales la fanega. La Gaceta de los Caminos de Hierro, n. ${ }^{\circ} 33,23$ de agosto de 1857, señalaba que el ferrado de trigo del gobierno en Galicia se estaba vendiendo a 10 reales. La fanega, por lo general, tiene cuatro ferrados.

67. Carta al Director de la Fábrica, 25 de agosto de 1857, AHA, PJAV. 
hemina de trigo de buena calidad de Castilla se estaba pagando a 11 reales y a 16 reales puesta en casa, lo que hacía que una fanega se pudiera adquirir a 48 reales, mientras que el copín de maíz se conseguía a $6,5^{68}$. Ahora bien, las fanegas de trigo expedidas por copinos se cobraban a los trabajadores a 84 reales y a 60 después de la rebaja de mediados del mes de agosto. Hay que precisar que los precios en los mercados regionales del trigo eran bastante buenos, ya que en el de Tordesillas se estaba pagando por la fanega de trigo nuevo una horquilla de precios entre $40 \mathrm{a}$ 44 reales y entre 42 y 46 reales en el de Santander, mejor comunicado desde el 24 de marzo de 1857, cuando se inauguró el tramo de Ferrocarril desde Alar de Rey, lugar donde concluía el Canal de Castilla ${ }^{69}$.

Por tanto, el Almacén de Granos de la Fábrica de Mieres no cumplió escrupulosamente con su propósito de vender al precio de coste sus artículos a los trabajadores. Así Ramón Martínez se quejaba al comerciante Juan Gómez de que el último maíz adquirido había tenido un coste total de 7 1/4 reales para la empresa, mientras que el precio al que se venía descontado al trabajador antes de la rebaja de agosto era de 8 reales el copín, es decir 25,5 maravedíes más caro que su verdadero precio de $\operatorname{coste}^{70}$. Sabemos además que durante el mes de julio se había conseguido comprar cargamentos de maíz incluso por debajo de los 55 reales la fanega y por tanto con menor coste final.

Hay que resaltar que los precios que logró la Fábrica de Mieres nunca fueron de los más baratos. Esta siempre tuvo un problema crónico de liquidez y nunca adelantó el capital necesario para adquirir los granos, como manifestaba el director en su declaración de intenciones. Todas las mercancías se abonaban mediante letras de cambio y nunca en efectivo ${ }^{71}$. Ahora bien, también parece que existieron problemas importantes para que los proveedores pudieran descontar las letras, como así se manifiesta en la carta de 23 de marzo de Ramón Martínez a Vicente Díaz, en la que le dice que el importe adeudado sería pagado con letras de cambio extrañamente giradas sobre las plazas de Valladolid y Bilbao a 8 días vista, manifestando expresamente que esto se hacía para que no fueran descontadas ${ }^{72}$. Parece ser que los problemas con los descuentos de las letras fueron corrientes por falta de credibilidad de la compañía. Como los apuros de tesorería continuaban a principios del mes de julio, ante la insistencia por parte de los proveedores, hubo que suscribir un préstamo con la casa de banca Herrero de Oviedo por más de 40.000 reales para hacer frente al pago de los granos adeudos ${ }^{73}$. Por otro lado, en la correspondencia del Almacén de Granos encontramos varias cartas en las que

68. 25 de agosto de 1857, AHA, PJAV. La hemina era una medida castellana que equivalía a un $1 / 3$ de fanega. 1857.

69. Gaceta de los Caninos de Hierro, n. ${ }^{\circ} 36,6$ de septiembre de 1857, y n. ${ }^{\circ} 32,16$ de agosto de

70. Carta a Juan Gómez de 21 de agosto de 1857, AHA, PJAV.

71. Cartas de 8 de febrero, 23 de marzo y 20 de agosto de 1857, AHA, PJAV.

72. Carta de Ramón Martínez a Vicente Díaz, 23 de marzo de 1857 AHA, PJAV.

73. Carta de 9 de julio de 1857, AHA, PJAV. 
D. GONZÁLEZ PALOMARES, L. A. GONZÁLEZ PRIETO Y J. MUÑIZ SÁNCHEZ

CARBÓN, HIERRO Y MAÍZ. ESTUDIO DE UN CASO PRECOZ DE TRUCK SYSTEM EN ESPAÑA: FÁBRICA DE MIERES (ASTURIAS), 1856-1857

los más importantes proveedores -los mayoristas Juan Valdés Hevia, Viuda de Jorge Díaz y Juan Gómez- exigían cobrar los granos suministrados hacía más de un mes, así como otras en las que se expresan las disculpas de los responsables del almacén por estos retrasos ${ }^{74}$.

El libro copiador de cartas del Almacén de Granos fecha su última misiva el 26 de agosto de 1857, no sabemos si porque se cerró el almacén o porque a partir de entonces las cartas se copiaron en otro cuadernillo que no hemos podido localizar. Posiblemente fuese una experiencia puntual que desapareció por no alcanzar las expectativas que se había propuesto de rebajar sustancialmente los precios de los cereales para sus empleados de una manera más filantrópica. Sin embargo, no debemos ignorar la crítica situación económica por la que en ese mes de diciembre de 1856 pasaba la Compagnie Minière et Métallurgique des Asturies. El Almacén de Granos y el truck system fueron sin duda unas medidas que aliviaron las necesidades de dinero de la empresa para pagar mensualmente los salarios de su gran cantidad de empleados.

En resumen, el Almacén de Granos consiguió para la empresa alargar en uno o dos meses el pago de una buena parte de los salarios de los obreros, en un momento en el que sus problemas económicos eran evidentes. A su vez logró evitar un eventual estallido de ira de los obreros por una hipotética falta de liquidación de sus salarios por estas dificultades de tesorería.

\section{CONCLUSIÓN}

El Almacén de Granos de la Fábrica de Hierro de Mieres -fundado en el mes de diciembre de 1856- es el primer comercio de empresa del que tenemos constancia no ya en Asturias, sino en toda España. Es el precedente más antiguo que conocemos del gran desarrollo que - a partir de finales del siglo xix y durante gran parte del xx- tuvieron los economatos laborales, que durante mucho tiempo fueron un canal importantísimo de distribución de productos de consumo para las clases populares. Esta tradición finalizó precisamente en el año 2017 con la venta de los establecimientos de la empresa HuNOsa a una cadena de alimentación privada. También constituye la primera referencia de la utilización del sistema del truck system por parte de una empresa industrial, que entrega cereales a cambio de vales a modo de adelanto sobre el sueldo.

Como se ha visto a lo largo de estas páginas, el año 1856 fue muy complicado desde el punto de vista social en Asturias. En un contexto en el que la fábrica de Mieres empezaba a tener un peso específico importante -con un número más que apreciable de obreros- y quizá en un intento de establecer un sistema de gestión del personal inspirado en las corrientes paternalistas que se desarrollaban por el continente, el director Fettyplace trató de poner en práctica una política social

74. Carta de Ramón Martínez a Vicente Díaz, 20 de agosto de 1857, AHA, PJAV. 
incipiente. Así, en ese año se fundó la Caja de Ahorros, a la que los trabajadores que lo deseaban -unos 160 - vertían un día de salario mensual y a cambio recibían medio jornal en caso de enfermedad además de atención médica y farmacéutica para su familia ${ }^{75}$. Pero el gran elemento de esta pretendida filantropía fue sin duda el establecimiento -por la vía de urgencia, dada la premura que marcaba la complicada situación de los abastos en la provincia- de Almacén de Granos del que se ha venido tratando. Las declaraciones a su constitución y sus primeros pasos parecían encaminados a tratar de aliviar la situación de agobiante necesidad en la alimentación de sus trabajadores, pero pronto giró hacia un sistema que en realidad no favorecía a sus obreros sino que se convirtió en un modo de financiar la falta de liquidez de la empresa para pagar los salarios, como se ha reseñado también.

Fue una medida que reportó importantes beneficios a la empresa, si no directamente mediante la imposición de precios abusivos o la venta de género de pésima calidad como ocurrió en otras empresas, sí por convertirse en una manera peculiar de financiar el capital circulante necesario para el pago de salarios. Esto permitió, en la situación de apuro económica en la que se encontraba la empresa en aquellas fechas, posponer un tiempo las perentorias necesidades de liquidez para este particular. Consecuentemente, debió de ayudar en gran medida a mantener una cierta paz social, ya que no contar con el numerario suficiente para la liquidación puntual del total de la nómina de los trabajadores hubiera probablemente sido motivo de motines y algaradas importantes. Si bien se trató de un caso coyuntural de una corta duración, su constitución y desarrollo se han fijado como un hito dentro de la historia industrial asturiana y española.

75. Vega, José Antonio: Historia de Fábrica de Mieres. El Mieres de la Fábrica: un ensayo documentalista. Oviedo: El sastre de los libros, 2017, p. 26. 